\title{
Serum level of Interleukin 29 in verruca vulgaris: Case control study
}

\section{Abdelraheem TA ${ }^{(1)}$, El-Tahlawi SM ${ }^{(2)}$, Shaker OG ${ }^{(3)}$, Mohamed MH $^{(4)}$, Amer MA $^{(5)}$, Farouk $\mathbf{N}^{(6)}$, Lotfi $\mathbf{W}^{(7)}$.}

(1) Professor of Dermatology, STDs and Andrology department, Faculty of Medicine, Fayoum University.

(2) Professor of Dermatology department, Faculty of Medicine, Cairo University.

(3) Professor of Biochemistry, Faculty of Medicine, Cairo university.

(4) Lecturer of Dermatology STDs and Andrology, Faculty of Medicine, Fayoum University.

(5) Lecturer of Dermatology, Faculty of Medicine, Cairo University.

(6) Ass. lecturer of Dermatology, Faculty of Medicine, Cairo University.

(7) Department of Dermatology, Faculty of Medicine, Fayoum University.

Corresponding author: Dr. wahid lotfi, E-mail: wahid_lotfi@yahoo.com

Fax: +2084636583

Tel: 01000978909

\begin{abstract}
Verruca vulgaris is a common cutaneous viral infection. It presents as hyperkeratotic, exophytic, dome-shaped papules or nodules often located on fingers, hands, knees, elbows, or sites of trauma. It is common in childern, young adults, and immunosuppressed individuals. IL-29 (also known as IFN-lamda1) has antiviral activities. IL-29 induces antiviral proteins such as MX1, BST2, ISG15, and OAS2 that increase the antiviral competence of keratinocytes.
\end{abstract}

The aim of this study is to detect the serum level of IL29 in patients infected with KEY WARDS: Verruca vulgaris, IL-29, ELISA.

\section{INTRODUCTION}

Warts are very common benign growths caused by HPV and can affect skin and mucous membranes. There are many clinical presentations of warts including verruca vulgaris, periungual and subungual verruca, plantar warts, plane warts, filiform warts and Butcher's wart [1].

Verruca vulgaris affects exposed surfaces, mainly the dorsal aspect of the hands verruca vulgaris . This may suggest a possible role of IL29 in HPV infection.

This study was in the period between October 2017 and February 2018. Forty patients with verruca vulgaris and forty healthy controls were included.

Our results show Serum level of IL-29 was higher in patients than in controls and this was statistically significant $(\mathrm{P}$ value $=0.001)$

Increase serum IL-29 level in patients with verruca vulgaris as a result of chronic infection with HPV and occurs as a defense mechanism against this infection. and fingers and presents as firm, rough keratotic verrucous papules [2].

IL-29 (also known as Interferon lambda 1) belongs to type III INF family which is secreted from maturing dendritic cells, Th17 cells, macrophages, mast cells, and alveolar cells and targets skin cells including keratinocytes and melanocytes and also hepatocytes [3]. 
In response to viral infection IL-29 binds to its receptor and stimulates the induction of antiviral proteins, such as BST2, OAS1 and

\section{PATIENTS AND METHODS}

The aim of this study is to detect a possible role of IL-29 in patients infected with verruca vulgaris by comparing the serum level of IL29 in both patients and controls. This study was conducted on forty patients with verruca vulgaris and forty healthy controls of different age groups more than 12 years and both sexes. All Patients were subjected to detailed history
MX1, that produce an antiviral state and its expression correlates with their level [4].

\section{RESULTS}

taking and complete general and dermatological examination. A $5 \mathrm{ml}$ peripheral venous blood sample was taken from all patients and controls under sterile conditions for assessment of serum level of IL-29. The serum level of IL-29 was measured using Human Interferon lambda-1, IFN-lambda1 ELISA Kit.

This study revealed that IL-29 level was significantly higher in patients compared to controls $(\mathrm{P}$ value $=0.001)$

\begin{tabular}{|c|c|c|c|}
\hline & Patients & Controls & p Value \\
\hline IL-29 $(\mathbf{p g} / \mathbf{m l})($ Mean \pm SD) & $5.12 \pm 4.68$ & $1.90 \pm 1.29$ & 0.001 \\
\hline
\end{tabular}

\section{DISCUSSION}

To our knowledge, this is the first study to evaluate the serum level of IL-29 in patients with verruca vulgaris.

Serum level of IL-29 was significantly higher in patients compared to controls.

The level and role of IL-29 were well studied in other viral infections especially HCV, HBV, HSV [5, 6, 7, 8].

Increased levels of IL-29 in serum and increased expression of this cytokine and its receptor in the liver were reported during chronic hepatitis $\mathrm{C}$ virus (HCV) infection. This supports the immunomodulatory action of IL-29 [7]. Similar findings were reported in other viral infections including HBV and HIV $[5,8]$.

IL-29 was found to have antiviral effects against $\mathrm{HCV}$ directly through activation of STAT pathway and induction of potential effector genes (ISGs) causing inhibition of HCV replication [9].

It acts also indirectly through dendritic cell-dependent expansion of regulatory $\mathrm{T}$ cells which have an anti-inflammatory action [7, $10]$.

Several clinical trials were reported using IL-29 as a potential therapeutic agent to eradicate $\mathrm{HCV}$ infection with good effects without producing hematological toxicity and with limited undesired side effects compared to regular interferon $[7,11,12]$.

Increased IL-29 expression in response to herpes simplex virus-1 (HSV-1) infection in the skin was also reported. A strong positive feedback signals in response to HSV-1 infection occur because HSV-1 infection activates IL-29 expression in macrophages and dendritic cells which strongly enhances HSV-1 induced cytokine gene expression and protein 
production leading to greatly enhanced antiviral response. A role for IL-29 in the treatment of HSV-1 infections was also suggested [13].

Strong elevation of antiviral proteins (MX1, BST2, ISG15 and OAS2) in psoriatic lesions correlated with increased level of serum IL-29 and this was explained by IL-29 production by $\mathrm{TH} 17$, one of the major players in psoriasis [14]. This may explain the difference in frequency of viral infections in psoriasis $(7 \%)$ compared to atopic dermatitis (30\%) where IL-29 is absent due to decreased level of TH17 and the ability of IL-4 to decrease the IL-29 production. These findings suggest that IL-29 seems to be responsible for the antiviral competence in psoriasis [15].

In addition to its antiviral role, IL-29 was found to also have anti-proliferative effect on keratinocytes in psoriasis [15] and in some malignancies as well as apoptotic and antiangiogenic effects [16].

It was reported that patients with gastric cancer who responded to chemotherapy had higher serum IL-29 level than patients who did not respond suggesting that the antiproliferative effects of IL-29 might contribute to the response to chemotherapy. They also found that patients without metastasis had higher serum IL-29 level than those with metastasis supporting anti-angiogenic

\section{LIMITATION}

Small number of patients enrolled in our study so we recommend Measurement of IL-29 and

\section{CONCLUSION}

In conclusion, the mean serum level of IL-

29 in verruca vulgaris patients was characteristics of IL-29. The anti-proliferative and apoptotic effects of IL-29 are due to stimulating phosphorylation of the Janus kinase/signal transducers and activators of transcription (JAK/STAT) pathways that result in growth inhibitory signals [16].

We hypothesize that the increased serum IL-29 level in our patients with verruca vulgaris occurred as a result of chronic infection with HPV in a similar way it occurred in chronic HCV infection and we suggest that the positive feedback loop observed in $\mathrm{HCV}$ and $\mathrm{HSV}-1$ infections that enhances IL-29 level may be responsible for the statistically significant difference between our patients and controls.

This response occurs as a defense mechanism against HPV infection since IL-29 has a proved and well documented antiviral activity mainly through production of antiviral proteins in skin.

IL-29 may also act by mechanisms in HPV infection other than the antiviral immunity. Since verruca vulgaris is a benign hyper proliferative growth, the antiproliferative and anti-angiogenic properties of IL-29 may also play a role in this HPV infection. However, further studies are required to demonstrate all the possible roles of IL-29 in verruca vulgaris.

AVP in serum and tissue of patients with verruca vulgaris.

significantly higher compared to healthy control. There is a strong correlation.

\section{REFERENCES}

[1] Habif, T. P., Chapman, M. S., Dinulos, J. G., \&Zug,K. A. (2017): Skin Disease E-Book: Diagnosis and Treatment. Elsevier Health Sciences; 203-240.

[2] Adisen, E., \& Onder, M. (2017): Acral manifestations of viral infections. Clin. Dermatol.; 35(1): 40-49.

[3] Lazear, H. M., Nice, T. J., \& Diamond, M. S. (2015): Interferon- $\lambda$ : immune functions at barrier surfaces and beyond. Immuni; 43(1): 15-28.

[4] Pasparakis, M., Haase, I., \& Nestle, F. O. (2014): Mechanisms regulating skin immunity and inflammation. Nature Reviews Immunology; 14(5): 289-301. 
[5] Ank, N., West, H., Bartholdy, C., Eriksson, K., Thomsen, A. R., \& Paludan, S. R. (2006): Lambda interferon (IFN- $\lambda$ ), a type III IFN, is induced by viruses and IFNs and displays potent antiviral activity against select virus infections in vivo. J. virol.; 80(9): 4501-4509.

[6] Melchjorsen, J., Siren, J., Julkunen, I., Paludan, S. R., \& Matikainen, S. (2006): Induction of cytokine expression by herpes simplex virus in human monocyte-derived macrophages and dendritic cells is dependent on virus replication and is counteracted by ICP27 targeting NF- $\mathrm{B}$ and IRF-3. J. Gen. Virol.; 87(5): 1099-1108.

[7] Dolganiuc, A., Kodys, K., Marshall, C., Saha, B., Zhang, S., Bala, S., \& Szabo, G. (2012): Type III interferons, IL-28 and IL-29, are increased in chronic HCV infection and induce myeloid dendritic cell-mediated FoxP3+ regulatory T cells. PloS one, 7(10), e44915.

[8] Rallón, N. I., Soriano, V., Naggie, S., Restrepo, C., McHutchison, J., Vispo, E., \& Benito, J. M. (2012): Impact of IL28B gene polymorphisms on interferon- $\lambda 3$ plasma levels during pegylated interferon- $\alpha$ /ribavirin therapy for chronic hepatitis $\mathrm{C}$ in patients coinfected with HIV. J. Antimicrob. Chemother.; 67(5): 1246-1249.

[9] Marcello, T., Grakoui, A., Barba-Spaeth, G., Machlin, E. S., Kotenko, S. V., Macdonald, M. R., $\&$ Rice, C. M. (2006): Interferons $\alpha$ and $\lambda$ inhibit hepatitis $C$ virus replication with distinct signal transduction and gene regulation kinetics. Gastroenterology; 131(6): 1887-1898.

[10] Kitching, A. R., \& Holdsworth, S. R. (2011): The emergence of TH17 cells as effectors of renal injury. J.A.S.N.; 22(2): 235-238.

[11] Donnelly, R. P., \& Kotenko, S. V. (2010): Interferon-lambda: a new addition to an old family. J INTERF CYTOK RES; 30(8): 555-564.

[12] Kelly, C., Klenerman, P., \& Barnes, E. (2011): Interferon lambdas: the next cytokine storm. Gut, gut-2011. B.M.J.; 60 (9):1284-1293.

[13] Melchjorsen, J., Siren, J., Julkunen, I., Paludan, S. R., \& Matikainen, S. (2006): Induction of cytokine expression by herpes simplex virus in human monocyte-derived macrophages and dendritic cells is dependent on virus replication and is counteracted by ICP27 targeting NF- $\mathrm{KB}$ and IRF-3. J. Gen. Virol.; 87(5): 1099-1108.

[14] Johnston, A. (2016): Interleukin-29: Just an extra string in the bow of Th17 cells or a target for therapeutic exploitation? J. Mol. Med.; 94(4): 373-376.

[15] Wolk, K., Witte, K., Witte, E., Raftery, M., Kokolakis, G., Philipp, S., \& Sterry, W. (2013): IL29 is produced by TH17 cells and mediates the cutaneous antiviral competence in psoriasis. Sci. Transl. Med.;5(204): 204ra129.

[16] Erturk, K., Tastekin, D., Serilmez, M., Bilgin, E., Bozbey, H. U., \& Vatansever, S. (2016): Clinical significance of serum interleukin-29, interleukin-32, and tumor necrosis factor alpha levels in patients with gastric cancer. Tumor Biol.; 37(1): 405-412. 